\title{
CONSTRUCCIÓN Y APLICACIÓN DE UNA FORMA DE MEDIDA DE LA DIVERSIFICACIÓN EMPRESARIAL
}

\section{CONSTRUCTION AND APPLICATION OF A MEASURE FORM OF THE MANAGERIAL DIVERSIFICATION}

\author{
Patricia Huerta Riveros ${ }^{1}$ \\ José Emilio Navas López ${ }^{2}$ \\ Recibido 9 de julio de 2007, aceptado 30 de noviembre de 2008 \\ Received: July 9, 2009 Accepted: November 30, 2008
}

Paloma Almodóvar Martínez ${ }^{2}$

\begin{abstract}
RESUMEN
Este artículo presenta, en primer lugar, un breve análisis crítico de las diferentes formas de medida de las estrategias corporativas que recoge la literatura especializada. Sin embargo, frente a la imposibilidad de poder medir la diversificación, a través de las formas tradicionales, se ha optado por la construcción y aplicación de una forma de medida, a partir de la información suministrada por la Encuesta sobre Estrategias Empresariales (ESEE). El periodo de análisis es del año 1991 hasta el año 2005 y se aplica la forma de medida propuesta a una muestra de empresas industriales españolas. Posteriormente, se realiza un análisis de la distribución de empresas diversificadas por sectores industriales, al igual que un análisis de la distribución de las empresas según su tamaño. Los resultados arrojan que en la realidad española sólo el $15 \%$ de las empresas siguen estrategias de diversificación y que la mayoría de las empresas se encuentran en la categoría de menor dimensión.
\end{abstract}

Palabras claves: Diversificación, diversificación relacionada, diversificación no relacionada, tamaño.

\section{ABSTRACT}

This article presents, in the first place, a brief critical analysis of the different ways of measure of the corporate strategy that the specialized literature has published. However, given to the difficulty of measuring the diversification construct through traditional forms, it has been chosen the construction and application of a way of measure starting from the information given by the Survey on Business Strategies (ESEE). The analysis period is from 1991 to 2005 and the form of measure proposal is applied to a sample of spanish industrial firms. Later on, we analyzes the distribution of diversified firms by industrial sectors and an analysis of the distribution of the firms according to their size. The results show that in the spanish case only $15 \%$ of the companies follows diversification strategies and that most of the companies fall into smallest dimension category.

Keywords: Diversification, related diversification, unrelated diversification, size.

\section{INTRODUCCIÓN}

La forma de medir la diversificación de la empresa es un problema relevante que ha interesado tanto a los académicos del área de la Dirección de Empresas como a los profesionales y ejecutivos responsables de su aplicación práctica. Son muchos los trabajos aparecidos en la literatura que proponen distintos instrumentos de medida de la variable diversificación, tanto relativos a la identificación del grado como de la dirección de la diversificación empresarial. Sin embargo, el acceso a la información apropiada y la dificultad para medir impiden identificar la estrategia corporativa fácilmente.
Por esta razón, el presente trabajo intenta contribuir con la literatura existente a partir de un breve análisis crítico de las diferentes formas de medidas sobre diversificación y a través de la propuesta de un índice de diversificación, construido a partir de la información disponible en la Encuesta sobre Estrategias Empresariales (ESEE). Este índice permite conocer tanto el grado como la dirección de la diversificación de las empresas industriales españolas, para el periodo 1991-2005. Además, para una mayor comprensión se realiza una desagregación del análisis que permite observar la distribución de las empresas diversificadas en función del tipo de sector y el tamaño de la empresa.

\footnotetext{
1 Departamento de Administración y Auditoría. Facultad de Ciencias Empresariales. Universidad del Bío-Bío. Avenida Collao 1202. Casilla 5-C. Concepción-Chile. E-mail: phuerta@ubiobio.cl

2 Departamento de Organización de Empresas. Facultad de Ciencias Económicas y Empresariales. Universidad Complutense de Madrid. Campus de Somosaguas, 28223-Pozuelo de Alarcón. Madrid, España. E-mail: jenavas@ccee.ucm.es, paloma.almodovar@ccee.ucm.es
} 


\section{TIPOS DE MEDIDAS DE DIVERSIFICACIÓN}

Como se acaba de exponer, es muy abundante la literatura sobre las formas de medida de la diversificación, ya que, como señalaba Rumelt [36], no están generalmente aceptadas ni la definición ni las medidas sobre el concepto de diversificación. En esta misma línea, Pitts y Hopkins [31] ponen de manifiesto que existen múltiples formas de medición, pero la elección de la medida debe ser guiada principalmente por la pregunta de investigación. De igual forma, Ramanujan y Varadarajan [33] realizan una revisión de los distintos enfoques utilizados para medir la diversificación empresarial, señalando que la utilización de unos u otros tipos de medidas depende de la pertenencia a distintas disciplinas del autor que las emplea (Economía Industrial, Finanzas, Dirección Estratégica). Del análisis de las medidas propuestas se pueden deducir tres criterios, que representan ópticas distintas de acercarse al mismo fenómeno, a partir de los cuales se pueden establecer diferentes tipologías de medidas sobre diversificación [30, 33]:

\section{1) El aspecto de la diversificación que se trata de medir:}

a) La extensión o el grado de diversificación en la empresa, es decir, la mayor o menor diversidad de su cartera de negocios [26]. b) La dirección de la diversificación, que identifica el nivel de relación existente entre los distintos negocios, según si éstos se encuentran relacionados o no relacionados [28]. c) El modo o método de diversificación, que pone de manifiesto la forma mediante la cual se hace la diversificación, según sea de crecimiento interno (inversiones que realiza la empresa) o externo (a través de fusiones y adquisiciones) [20].

\section{2) El enfoque adoptado para la medición:}

a) Medición objetiva: basada en criterios objetivos, sin introducir juicios de valor sobre el objeto de estudio por parte del investigador. b) Medición subjetiva: basada en la utilización de criterios o juicios de valor por parte del investigador.

\section{3) Los niveles de las medidas utilizadas:}

a) Medidas continuas: a través de variables continuas que miden distintos aspectos (extensión, dirección o método) de la diversificación. b) Medidas categóricas: basadas en la definición de categorías de estrategias sobre las cuales se establecen criterios para poder incluir una empresa en una de las categorías.

De las tres clasificaciones anteriores, la más desarrollada es la tercera que diferencia entre medidas continuas y medidas categóricas [38], en buena medida, porque incorpora implícitamente algunos de los aspectos considerados en las otras dos categorías. A continuación, se realiza un breve análisis comparado para conocer sus ventajas e inconvenientes.

Medidas continuas: Las medidas continuas se basan en los sistemas de clasificación industrial propuestos por distintos organismos, tanto públicos como privados, y tratan de clasificar la actividad de cada empresa dentro de un sector industrial. Estos organismos son ajenos a la empresa, lo que garantiza la objetividad de la medida. Específicamente, en España el sistema de clasificación industrial que se emplea es la Clasificación Nacional de Actividades Económicas (CNAE) ${ }^{3}$, la cual sigue las pautas de la clasificación industrial internacionalmente más conocida, como es la Clasificación Industrial Estándar (SIC), que clasifica a todos los productos o servicios de la economía de Estados Unidos.

Las principales ventajas que presentan estas medidas son: a) Su naturaleza objetiva y la facilidad de cálculo o medición (donde ambas ventajas se deben a que se basan en una clasificación ya establecida), b) El menor tiempo requerido y el costo para la clasificación de la empresa, puesto que no se requiere observar y analizar demasiada información de cada empresa [16].

Sin embargo, no están exentas de limitaciones. Específicamente, es criticable la definición de las distintas actividades a la que da lugar, y aunque permiten distinguir entre diversificación relacionada y no relacionada, agrupan los productos por similaridades en función de la tecnología productiva o en función de cuáles sean sus principales inputs [16]. Por lo tanto, sólo considera las relaciones existentes de tipo productivo o, en todo caso, tecnológicas.

Estas limitaciones se basan en los siguientes argumentos: a) en primer lugar, las reglas para la definición del sector no están perfectamente definidas, y si bien prevalece un criterio de oferta, basado en la similitud de procesos productivos (en la medida en que los datos se recogen para empresas o establecimientos), también se debería

\footnotetext{
3 En España se emplea como criterio de sectorización la CNAE-93, Clasificación Nacional de Actividades Económicas, según el Real Decreto 1560/1992, del 18 de diciembre. Ésta actualiza a la CNAE74 y permite dar cumplimiento a lo dispuesto en el Reglamento del Consejo de la Comunidad Económica Europea número 3037/1990, del 9 de octubre, por el que se establece la nomenclatura estadística de actividades económicas en la Comunidad Europea, denominada NACE (Revisión 1) de utilización obligatoria para todas las estadísticas oficiales de actividades económicas de los países comunitarios. Además, sobre esta base se elabora la Contabilidad Nacional y las tablas Input-Output por el Instituto Nacional de Estadística [3].
} 
emplear un criterio de demanda; b) en segundo lugar, la precisión con que está definido el producto no tiene por qué ser siempre idéntica, aun dentro del mismo nivel de desagregación, por lo que el grado de "amplitud" en cada sector es distinto. Además, implícitamente se supone la misma desigualdad entre unos sectores y otros, si bien este problema se atenúa en la medida en que, al utilizar distintos niveles de agregación, se consideran más próximos aquellos sectores que se fusionan en un nivel de agregación superior, y c) las distancias entre los sistemas de códigos de clasificación industrial no pueden medirse mediante ningún ratio o escala de intervalo. Por ejemplo, la distancia entre el refino de petróleo y la industria química se supone igual que la distancia entre la industria química y la de materiales de construcción, o las actividades agrícolas y ganaderas igual que la ganadera y el comercio al por menor [40].

Sin embargo, a pesar de los problemas que siempre incorpora la utilización de clasificaciones sectoriales para la valoración de la diversificación, su uso es generalizado, ya sea por su objetividad, por ser una nomenclatura estándar y por su disposición o acceso a tal clasificación.

Por último, este tipo de medidas que tuvo su mayor aplicación entre autores pertenecientes al campo de la Economía Industrial, también han sido utilizadas en trabajos desarrollados desde la perspectiva de la Dirección Estratégica ${ }^{4}$. Esta última perspectiva, pese a que utiliza de forma predominante el concepto de negocio y no el de mercado, también hace uso de las clasificaciones sectoriales para dotar de cierta superioridad a la medición de la diversificación [22]. Por ello, Pehrsson [29] plantea que los investigadores han evaluado la relacionalidad entre las diferentes unidades de negocios a través de la comparación de códigos de negocios SIC o por el uso de ciertos índices. Dentro de esta perspectiva, la investigación estratégica sobre diversificación a menudo excluye a las empresas de un único negocio o empresas especializadas, porque las medidas de relacionalidad son definidas sólo para empresas multinegocios [24]. La tabla 1 recoge las principales ventajas e inconvenientes de las medidas continuas. En concreto, entre las medidas continuas más conocidas se encuentran el índice de contabilización de productos (Índice de Berry-Herfindahl), el índice de entropía [27] y el espectro de diversidad de Varadarajan y Ramanujam [44], cuyas características básicas se encuentran recogidas en la tabla 4.

\footnotetext{
4 La Dirección Estratégica es una disciplina relativamente joven. Dependiendo de la métrica utilizada está entre dos a tres décadas de edad, sin embargo, incluso con esta juventud, juega un rol crítico en el estudio de los negocios y de los gerentes [2].
}

Medidas categóricas: En cambio, las medidas categóricas fueron desarrolladas principalmente por especialistas en el área de estrategia empresarial, son de naturaleza cualitativa y además permiten distinguir el tipo de estrategia de diversificación que poseen las empresas. Es una tipología de carácter subjetivo (aunque también involucra medidas objetivas) que trata de recoger no sólo el grado de diversificación de una empresa sino también el tipo de relación que existe entre los negocios de la misma. En esencia, esta tipología de medición tiene su origen en la propuesta de Wrigley [47], posteriormente ampliada por Rumelt [36], y ha dado lugar a las categorías estratégicas de Wrigley/Rumelt. Esta clasificación se basa en la utilización de tres ratios:

a) Ratio de especialización productiva (RE): Es el porcentaje de la cifra total del negocio o de los ingresos de la empresa que corresponden a la actividad principal, es decir, aquella actividad en la que la empresa obtiene la mayor parte de sus ingresos $[9,12,36,40]$.

b) Ratio de relación ( $R R$ ): Es el porcentaje de la cifra total del negocio o de los ingresos de la empresa que corresponden al mayor grupo de negocios relacionados principales que posee la empresa [36].

Es decir, una vez que se ha distinguido entre empresas diversificadas y no diversificadas ${ }^{5}$ (a través del ratio de especialización) se analizan los distintos negocios que componen la cartera de las empresas diversificadas tratando de detectar posibles relaciones o similitudes entre ellos y estableciendo, en su caso, grupos de negocios relacionados. Por lo tanto, el ratio de especialización muestra el porcentaje de ventas correspondiente al mayor de estos grupos o es el porcentaje de ventas atribuido al grupo principal de negocios relacionados ${ }^{6}[9,12,40]$.

c) Ratio vertical (RV): Representa la proporción de las ventas o ingresos de la empresa que surgen (de todos los productos, productos intermedios y productos finales de una secuencia de actividades integradas verticalmente) atribuidas a la cadena de integración vertical $[12,36]$.

La clasificación de Wrigley/Rumelt se encuentra recogida en la tabla 3 .

\footnotetext{
5 En la actualidad la distinción entre diversificación relacionada y no relacionada se puede entender como la vinculación entre las líneas de negocios que posee una empresa a través de las habilidades, recursos, mercados y propósitos comunes [7].

6 El ratio de relación suele incluir a la actividad principal (con lo cual el RE quedaría contenido en RR), sin embargo, en teoría no tiene por qué ser así. En efecto, puede darse el caso en que haya un conjunto de actividades secundarias relacionadas entre sí pero sin relación alguna con la principal [40].
} 
Sin embargo, para comprender mejor esta clasificación, en cuanto a los valores de los distintos ratios, conviene revisar los puntos de corte utilizados originariamente por Wrigley [47], los cuales se basan en la existencia de una bimodalidad en la distribución de los ratios de especialización de las empresas estudiadas. En concreto, su propuesta original definía los siguientes tipos de negocios:
- Negocio Único (NU): RE $\geq 95 \%$.

- Negocio Dominante (ND): $70 \% \leq \mathrm{RE}<95 \%$.

- Diversificación Relacionada (DR): RE $<70 \%$ y RR $\geq 70 \%$.

- Diversificación No Relacionada (DNR): RE $<70 \%$ $\mathrm{y} \operatorname{RR}<70 \%$.

Tabla 1. Ventajas e inconvenientes de las medidas continuas.

\begin{tabular}{|c|c|c|c|}
\hline Ventajas & Autores & Inconvenientes & Autores \\
\hline - Objetividad & $\begin{array}{l}{[12,14,19,} \\
21,25,27, \\
33,40,44]\end{array}$ & $\begin{array}{l}\text { - Es criticable la definición de las distintas } \\
\text { actividades a las que da lugar el código } \\
\text { SIC }\end{array}$ & {$[21,40]$} \\
\hline $\begin{array}{l}\text { - Se basa en el código SIC para determinar } \\
\text { la diversidad de la empresa }\end{array}$ & $\begin{array}{l}{[12,21,25,} \\
27,37,38]\end{array}$ & $\begin{array}{l}\text { - Las distancias entre los códigos SIC se } \\
\text { miden mediante ratio o escala de intervalo } \\
\text { con igual distancia entre los códigos SIC } \\
\text { adyacentes }\end{array}$ & $\begin{array}{l}{[25,12,} \\
40]\end{array}$ \\
\hline $\begin{array}{l}\text { - Acceso rápido a la información disponible } \\
\text { (SIC) }\end{array}$ & {$[27,44]$} & $\begin{array}{l}\text { Sólo considera relaciones existentes de tipo } \\
\text { productivo o tecnológicas }\end{array}$ & {$[21,40]$} \\
\hline - Fácil de calcular o medir & {$[25,27]$} & Prevalece un criterio de oferta & {$[22]$} \\
\hline - Simplicidad de las Medidas (SIC) & {$[27,38]$} & \multirow[t]{5}{*}{ Imprecisión en la definición del producto } & \multirow[t]{5}{*}[22]{} \\
\hline $\begin{array}{l}\text { - Si dos negocios comparten el mismo código } \\
\text { SIC, ellos deben poseer requerimientos de } \\
\text { input en común, producciones similares y } \\
\text { funciones de tecnologías comunes }\end{array}$ & {$[21]$} & & \\
\hline - Menor tiempo requerido para el análisis & {$[25,38]$} & & \\
\hline $\begin{array}{l}\text { - Mide la extensión o el grado de } \\
\text { diversificación }\end{array}$ & {$[9,33]$} & & \\
\hline - Replicables & {$[27,37]$} & & \\
\hline
\end{tabular}

Tabla 2. Ventajas e inconvenientes de las medidas categóricas.

\begin{tabular}{|c|c|c|c|}
\hline Ventajas & Autores & Inconvenientes & Autores \\
\hline $\begin{array}{l}\text { - Categoriza la extensión y el tipo de } \\
\text { diversificación de las empresas }\end{array}$ & [44] & $\begin{array}{l}\text { - Subjetividad: El proceso de clasificación } \\
\text { requiere de juicios del investigador para } \\
\text { definir los negocios de la empresa y el grado } \\
\text { de relación y proximidad entre ellos }\end{array}$ & $\begin{array}{l}{[12,14,} \\
21,25,31, \\
33,38,40, \\
44]\end{array}$ \\
\hline $\begin{array}{l}\text { - Su naturaleza cualitativa permite distinguir } \\
\text { el tipo de estrategia de diversificación }\end{array}$ & [40] & - El tiempo requerido es mayor & $\begin{array}{l}{[12,25,31,} \\
44]\end{array}$ \\
\hline $\begin{array}{l}\text { - Se basa sobre la lógica fundamental que hay } \\
\text { detrás de las intenciones de la empresa y } \\
\text { la relación entre las unidades de negocio }\end{array}$ & {$[36,38]$} & $\begin{array}{l}\text { - Se requieren varias fuentes de información: } \\
\text { Desventaja en inversión de información } \\
\text { requerida para realizar el proceso de } \\
\text { categorización de Rumelt }\end{array}$ & {$[25,44]$} \\
\hline $\begin{array}{l}\text { - Los negocios son relacionados si poseen } \\
\text { habilidades, fortalezas, recursos, mercados } \\
\text { o aplican propósitos en común. Intentos } \\
\text { tangibles por explotar alguna característica } \\
\text { común }\end{array}$ & $\begin{array}{l}{[21,36,} \\
38]\end{array}$ & - La fiabilidad es cuestionable & {$[12,25]$} \\
\hline
\end{tabular}


Tabla 3. Resumen de los principios de clasificación de Wrigley /Rumelt.

\begin{tabular}{|l|l|l|l|}
\hline \multicolumn{1}{|c|}{ Categorías } & \multicolumn{1}{|c|}{ Ratio de especialización } & Ratio vertical & \multicolumn{1}{c|}{ Ratio de relación } \\
\hline Negocio Único & $\mathrm{RE} \geq 95 \%$ & & \\
\hline Vertical Dominante & & $\mathrm{RV} \geq 70 \%$ & \\
\hline Negocio Dominante: & $95 \%<\mathrm{RE} \leq 70 \%$ & $\mathrm{RV}<70 \%$ & \\
\hline - Dominante Limitado & $95 \%<\mathrm{RE} \leq 70 \%$ & & \\
\hline - Dominante Vinculado & $95 \%<\mathrm{RE} \leq 70 \%$ & & \\
\hline - Dominante No Relacionado & $95 \%<\mathrm{RE} \leq 70 \%$ & & $\mathrm{RR}<0,5(\mathrm{RE}+1)$ \\
\hline Negocio Relacionado: & $\mathrm{RE}<70 \%$ & $70 \%<\mathrm{RV}$ & $\mathrm{RR} \geq 70 \%$ \\
\hline - Relacionado Limitado & $\mathrm{RE}<70 \%$ & & \\
\hline - Relacionado Vinculado & $\mathrm{RE}<70 \%$ & & $\mathrm{RR}>70 \%$ \\
\hline Negocios No Relacionados & $\mathrm{RE}<70 \%$ & $\mathrm{RR}<70 \%$ \\
\hline
\end{tabular}

Fuente: Adaptado de Montgomery [25], Grant y Jammine, [9], Hall y John, [12].

Tabla 4. Medidas de diversificación.

\begin{tabular}{|c|c|c|}
\hline Medida & Autor & Fórmula /Descripción \\
\hline $\begin{array}{l}\text { Contabilización de productos } \\
\text { (Índice de Berry-Herfindahl } \\
\text { modificado) }\end{array}$ & {$[25]$} & 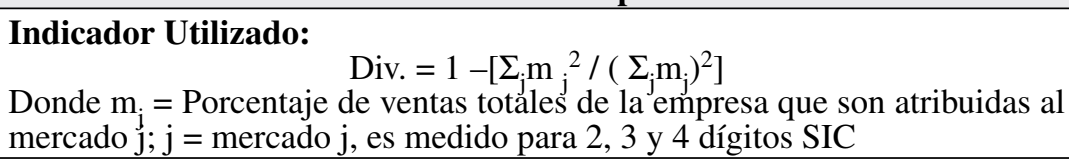 \\
\hline Índice de entropía & [27] & $\begin{array}{c}\begin{array}{c}\text { Indicador utilizado: } \\
\boldsymbol{D T}=\boldsymbol{D R}+\boldsymbol{D N R \boldsymbol { R }}\end{array} \\
\mathrm{DT}=\sum_{\mathrm{j}=1}^{\mathrm{M}} \mathrm{DR}_{\mathrm{j}} \mathrm{P}^{\mathrm{j}}+\sum_{\mathrm{j}=1}^{\mathrm{M}} \mathrm{P}^{\mathrm{j}} \ln \left(\mathrm{l} / \mathrm{P}^{\mathrm{j}}\right) \\
\text { Donde } \mathrm{M}=\text { Número de grupos de industria; } \mathrm{j}=1 \ldots, \mathrm{M} ; \mathrm{P}^{\mathrm{j}}=\text { Proporción de las } \\
\text { ventas del grupo j-ésimo, en el total de ventas de la empresa }\end{array}$ \\
\hline $\begin{array}{l}\text { Espectro de diversidad } \\
\text { amplio (extenso) o estrecho } \\
\text { (reducido) }\end{array}$ & [44] & $\begin{array}{l}\text { Indicadores utilizados: } \\
\text { BSD: número de códigos de dos dígitos SIC en el cual una empresa } \\
\text { participa } \\
\text { MNSD: número de códigos de cuatro dígitos SIC en el que una empresa } \\
\text { participa dividido por el número de categorías de dos dígitos SIC en que la } \\
\text { empresa opera }\end{array}$ \\
\hline $\begin{array}{l}\text { Clasificación de Wrigley/ } \\
\text { Rumelt }\end{array}$ & {$[36,47]$} & $\begin{array}{l}\text { Indicadores utilizados: } \\
\text { Ratio de especialización (RE); Ratio de Relación (RR); Ratio Vertical (RV) } \\
\text { Categorías definidas: Negocio Único; Negocio Vertical Dominante; Negocio } \\
\text { Dominante; Negocio Relacionado; Negocio No Relacionado }\end{array}$ \\
\hline
\end{tabular}

Fuente: Adaptado de Sambharya [38].

Es decir, Wrigley [47] observó que las empresas optan por diversificarse en gran medida o por no hacerlo apenas (por ello, la mayoría de las empresas presentan una ratio de especialización por encima del $80 \%$ o por debajo del 60\%). De esta forma, la existencia de una cierta discontinuidad en el intervalo $80-60 \%$ es el argumento utilizado para fijar el 70\%, es decir, la línea de demarcación entre las empresas diversificadas y las especializadas en una $\operatorname{actividad}^{7}[40]$.

7 Algunos autores han criticado la falta de argumentos técnicos que sustenten la elección de estos puntos de corte [45]. Sin embargo, Reed y Sharp [35] aportan nueva evidencia empírica a la existencia de esa bimodalidad en las empresas inglesas y con ello validan estos
No obstante, el principal inconveniente de esta forma medida es su subjetividad [33], ya que requiere la introducción de juicios por parte de la persona que clasifica, tanto para definir cuáles son los negocios de la empresa como el grado de relación o proximidad existente entre ellos, lo cual puede ocasionar que diferentes investigadores pueden llegar a clasificar a las empresas de distinta forma [31].

Además, algunos investigadores han cuestionado la "fiabilidad" de las asignaciones categóricas de Rumelt, ya

puntos de corte utilizados tradicionalmente. Por último, Reed [34]
posteriormente desarrolla una argumentación teórica que explica la posteriormente desarrolla una argumentación teórica que
existencia de la bimodalidad en la diversificación [40]. 
que aunque la metodología tiene la fortaleza de realizar consideraciones de datos de la empresa, tanto cualitativas como cuantitativas, esta fortaleza podría volverse una debilidad si los criterios categóricos de un investigador no pudieran ser sistemáticamente reproducidos [25]. Es decir, la consideración de estos tipos de datos es difícil de aplicar en la práctica y están sujetos a preguntas acerca de la fiabilidad y las clasificaciones específicas del investigador [12].

A estos inconvenientes se podrían añadir otros como el tiempo consumido y el conjunto de datos necesarios, que requiere de numerosas fuentes de información para realizar tal clasificación, como, por ejemplo, informes anuales y otras publicaciones [44].

Pese a lo anterior, esta metodología, que ha sido principalmente utilizada por autores pertenecientes al área de Dirección Estratégica, representó un significativo avance conceptual sobre las medidas continuas, presentando una mayor riqueza y capacidad explicativa. Y, además, con ella se consigue establecer distintas categorías dentro de la estrategia de diversificación que identifican tanto su extensión como su dirección, a través de los valores tomados por las ratios establecidas. Ver tabla 2.

Como conclusión de este apartado podríamos señalar que algunos autores como Sambharya [38] consideran que una sola medida no es capaz de capturar todos los matices de cualquier estrategia de diversificación. Por esta razón, varios estudios han sugerido la utilización de múltiples medidas en la medición de variables estratégicas $[39,45]^{8}$.

Específicamente, los investigadores interesados en examinar la relación entre diversificación y desempeño han intentado desarrollar medidas de diversificación que combinen el atractivo conceptual del esquema de Rumelt [36] con la relativa objetividad y el acceso rápido a la información basados en el sistema de clasificación industrial [44] ${ }^{9}$.

Por ejemplo, Palepu [27] utiliza el índice de entropía de Jacquemin y Berry [18] para construir cuatro categorías de diversificación [44] y distingue entre diversificación relacionada y no relacionada. A su vez, Suárez González

8 Por ejemplo, los estudios de Hoskisson y Hitt [13] y Ramanujan y Varadarajan [33] animaron a los investigadores a utilizar múltiples medidas de diversificación para establecer la robustez de sus descubrimientos.

9 Para un análisis comparativo de las distintas formas de medida ver Huerta y Navas [15]: "Grado y dirección de la diversificación de las empresas industriales españolas: Un análisis de la estrategia de diversificación relacionada".
[40] utiliza los códigos CNAE para definir los distintos negocios de cada empresa y el grado de relación entre ellos, y posteriormente utiliza la clasificación de Wrigley/ Rumelt para establecer categoría de diversificación de cada empresa.

\section{APLICACIÓN EN LAS EMPRESAS INDUSTRIALES ESPAÑOLAS}

\section{Construcción de un índice de medición de la diversificación}

Debido a los inconvenientes de las distintas formas de medida antes analizadas, para captar simultáneamente el tipo y la dirección de diversificación seguido por las empresas, para la realización de este trabajo se optó por elaborar un índice propio que identifica empresas especializadas (es decir, no diversificadas), empresas con diversificación relacionada y empresas con diversificación no relacionada. Este índice se definió a través de los códigos de clasificación industrial de la Clasificación Nacional de Actividades Económicas (CNAE).

Para la elaboración de esta forma de medida se utilizó la información suministrada por la Encuesta sobre Estrategias Empresariales (ESEE).

Sin embargo, la ESEE, por razón de secreto estadístico, sólo ofrece información sobre diversificación empresarial a nivel de dos dígitos CNAE. Ante esta limitación se optó por intentar estimar unos indicadores más robustos utilizando tres dígitos de códigos CNAE, tal y como se recoge en la tabla 5, para identificar tanto el tipo como la dirección de la diversificación. Para ello se contó con la colaboración de la Fundación SEPI (Sociedad de Participaciones Industriales). Esta forma de medida es una transformación de datos ya validados por la ESEE, lo cual garantiza la validez del índice de diversificación utilizado.

\section{Determinación de la población y la muestra}

La población de referencia que se utilizó en la investigación fueron las empresas industriales españolas que reúne la ESEE, siendo el período elegido desde el año 1991 al 2005. La ESEE alcanza un alto grado de representatividad del conjunto de la industria española porque recoge datos de empresas ubicadas en veinte sectores industriales distintos. Específicamente, para la determinación de la muestra se partió de 47.103 observaciones de empresas industriales españolas, considerando el período de análisis de quince años. 
Tabla 5. Índices creados a partir de la ESEE.

\begin{tabular}{|c|l|l|}
\hline Índice & \multicolumn{1}{|c|}{ Estrategia } & \multicolumn{1}{c|}{ Criterio } \\
\hline 0 & No diversifica & $\begin{array}{l}\text { La empresa no está diversificada (sólo define un producto a 3 dígitos de códigos } \\
\text { CNAE) }\end{array}$ \\
\hline 1 & $\begin{array}{l}\text { Diversificación } \\
\text { relacionada }\end{array}$ & $\begin{array}{l}\text { La empresa tiene diversificación relacionada (define más de un producto a 3 dígitos, } \\
\text { y éstos están en el mismo sector a 2 dígitos). Ejemplo: 263, 264, 265 }\end{array}$ \\
\hline 2 & $\begin{array}{l}\text { Diversificación } \\
\text { no relacionada }\end{array}$ & $\begin{array}{l}\text { La empresa tiene diversificación no relacionada (define más de un producto a 3 dígitos, } \\
\text { y algunos de éstos están en distinto sector a 2 dígitos). Ejemplo: 263, 264, 325 }\end{array}$ \\
\hline
\end{tabular}

Tabla 6. Delimitación de la muestra.

\begin{tabular}{|c|}
\hline Número de observaciones de empresas para el período 1991-2005 \\
\hline 47.103 Observaciones de empresas en la base de datos de la ESEE en el período 1991-2005 \\
\hline-20.487 Observaciones de empresas que no responden a la ESEE en el período 1991-2005 \\
\hline 26.616 Observaciones de empresas que responden a la ESEE en el período 1991-2005 \\
\hline-1.884 Observaciones de empresas con valores perdidos en índice para el período 1991-2005 \\
\hline 24.732 Observación total de empresas que responden a la ESEE en el período 1991-2005 \\
\hline- 21.056 Observaciones de empresas que no diversifican en el período 1991-2005 \\
\hline 3.676 Muestra final de observaciones de empresas diversificadas desde 1991 hasta 2005 \\
\hline
\end{tabular}

No obstante, en la determinación de la muestra se excluyeron del estudio aquellas empresas que no diversifican, al igual que las empresas que no responden a la encuesta ${ }^{10}$. Con esto se pretendió reunir un conjunto de "observaciones de empresas diversificadas" para el horizonte temporal considerado en el estudio (1991-2005). Con ello se llegó a la identificación de 3.676 observaciones de empresas diversificadas, como se observa en la tabla 6 .

\section{Resultados del estudio}

Como se puede observar, del total de empresas que responde a la ESEE en el período considerado, es decir, 24.732 observaciones de empresas, sólo el $15 \%$ representa a las empresas diversificadas. Por lo tanto, podemos extraer una primera e importante conclusión del estudio al señalar que la gran mayoría de las empresas industriales españolas no diversifican. Además, de acuerdo con los datos de la ESEE, el 85\% de la población corresponde

\footnotetext{
${ }^{10}$ La ESEE entrega una variable control denominada IDSIT, la cual es una variable categórica que caracteriza la situación de la empresa en el año correspondiente. IDSIT toma cuatro valores: Sin acceso $(0)=$ 17.836, Responde $(1)=26.616$, Desaparece $(2)=827$ y, No colabora $(3)=648$. Además, esta variable presentó 1.176 observaciones perdidas, lo que generó 20.487 observaciones de empresas que no responden a la ESEE en el periodo 1991-2005.
}

a empresas especializadas que no desarrollan procesos de diversificación. Estos resultados son comprensibles si consideramos la tendencia en la actualidad hacia la reestructuración empresarial. Resultados similares a los obtenidos por Bueno, Morcillo, de Pablo [4] y Suárez González [40] quienes a partir de una muestra de empresas no financieras de la Central de Balances del Banco de España obtuvieron que el $82,7 \%$ y el $87 \%$ de las empresas no estaban diversificadas. Al igual, similares son los resultados obtenidos por Merino, de Lucas y Rodríguez [22], quienes realizan un análisis de la industria manufacturera española para el período 1990-1996, atendiendo a seis tramos de tamaño, encontrando que un porcentaje de empresas que oscila entre el 70 y $90 \%$ no diversifican cuando se utiliza la clasificación sectorial a tres dígitos CNAE, pero se reduce a 55-80\% cuando se considera la desagregación a cinco dígitos CNAE. En conclusión, estos resultados obtenidos muestran que la gran mayoría de las empresas manufactureras españolas no diversifican y, además, representan una actualización de los estudios antes mencionados.

La tabla 7 presenta el número total de observaciones de empresas especializadas y diversificadas y, la tabla 8 presenta una desagregación de las observaciones de empresas con diversificación relacionada y no relacionada. 
Específicamente, se puede apreciar que de la muestra 1.526 observaciones corresponden a empresas con diversificación relacionada y 2.150 observaciones corresponden a empresas con diversificación no relacionada. Para un mayor detalle, la tabla 8 también presenta los porcentajes que representan las empresas de la muestra según su dirección. En definitiva, los datos de esta tabla reflejan una ligera mayoría de empresas que se inclinan por la diversificación no relacionada frente a la relacionada. En efecto, el $58 \%$ de las empresas diversificadas optan por la no relacionada, lo que parece indicar que en la realidad española priman los objetivos de distribución del riesgo entre los distintos negocios frente a la creación de sinergias en actividades cercanas. Sin embargo, este resultado se debe observar con cierta cautela porque se puede deber a la forma en la cual hemos medido la diversificación. Por una parte, las empresas que presentaban ambas estrategias han sido clasificadas como no relacionadas para mostrar una mayor robustez en la categoría de diversificación relacionada. Por otra, es necesario recordar que al utilizar como forma de medida el sistema de clasificación industrial, éste sólo considera las relaciones existentes de tipo productivo o tecnológico y no otro tipo de relaciones (por ejemplo, canales de distribución, recursos estratégicos, capacidades, etc.) que pueden ser la esencia de la estrategia de diversificación relacionada.

Las tablas 9 y 10 muestran la desagregación de las observaciones de las empresas de la muestra para los veinte sectores industriales que contempla la ESEE, para las empresas diversificadas, empresas relacionadas y empresas con diversificación no relacionada, respectivamente.

En la tabla 9 se puede apreciar la media de las empresas en el sector industrial, la media de las empresas diversificadas, los porcentajes de empresas diversificadas en el sector y los porcentajes de empresas diversificadas en relación con los sectores manufactureros. En concreto, se observa que los sectores más diversificados son: el sector de productos metálicos, maquinaria y equipo mecánico, productos químicos, maquinaria y material eléctrico y electrónico, textiles y productos alimenticios y tabaco, cuyas medias son 33, 27, 22, 22, 21 y 18, respectivamente. En estos sectores se observa una mayor tendencia diversificadora histórica, que se aprecia a lo largo del periodo bajo estudio, debido quizás a una mayor complejidad tecnológica que incrementa la probabilidad de desarrollar sus productos y servicios en otros mercados.

A la vez, la literatura que explica las causas que llevan a una empresa a diversificar enfatizan la importancia que algunos de los rasgos ligados al tipo de actividad realizada pueden tener a la hora de determinar la adopción de una estrategia de diversificación, las características propias del sector y el tipo de tecnología empleadas con respecto al sector en el que se diversifica [22].

Como lo planteado por Tanriverdi y Lee [41], quienes señalan que la diversificación relacionada es especialmente prevalente en industrias que poseen alta tecnología. Otras causas mencionadas en la literatura son la reducción del riesgo global, la saturación del mercado tradicional, las oportunidades de inversión de los excedentes financieros y la explotación de sinergias [3], entre otras. Sin embargo, los beneficios de la diversificación también pueden disminuir debido al incremento en la complejidad directiva, estructural y organizativa [5]. Pese a lo anterior el camino que siga la empresa siempre debe buscar la creación de valor del cliente [43].

De la tabla 9 también se pueden observar los sectores industriales cuyo porcentaje de empresas diversificadas son mayores. Dentro de esta categoría se encuentran: el sector de máquinas de oficina, equipos informáticos, de proceso, óptica y similares, el sector de maquinaria y equipo mecánico, el sector de productos metálicos, el sector de metalurgia, el sector de maquinaria y material eléctrico y electrónico y el sector de productos químicos.

A la vez, también se ha analizado el peso de las empresas diversificadas, para lo cual se ha considerado cada sector industrial en relación con el total de las empresas diversificadas en la industria manufacturera para el periodo bajo estudio. De este análisis se ha descubierto que el mayor peso lo presentan los siguientes sectores: el sector de productos metálicos, el sector de maquinaria y equipo mecánico, el sector de productos químicos, el sector de maquinaria y material eléctrico y electrónico, el sector textil y el sector de productos alimenticios y tabaco.

Para complementar este análisis se ha realizado una desagregación de las empresas diversificadas, considerando en esta ocasión su dirección, relacionada y no relacionada. Información que se aprecia en la tabla 10. Los resultados obtenidos son los siguientes: los sectores industriales que presentan un mayor porcentaje de empresas con diversificación relacionada son: sector de bebidas y el sector de productos alimenticios y tabaco y el sector de productos químicos. En cambio, los sectores que presentan un mayor porcentaje de empresas con diversificación no relacionada son: el sector de máquinas de oficina, equipos informáticos, de proceso, óptica y similares, el sector de otro material de transporte, el sector de productos de caucho y plástico y el sector de muebles. 
Tabla 7. Desagregación de la muestra.

\begin{tabular}{|c|l|l|}
\hline Índice & \multicolumn{1}{|c|}{ Estrategia } & \multicolumn{1}{c|}{$\mathbf{N}^{\mathbf{0}}$ de observaciones 1991-2005 } \\
\hline 0 & No diversifica & 21.056 \\
\hline 1 & Diversificación relacionada & 1.526 \\
\hline 2 & Diversificación no relacionada & 2.150 \\
\hline $1+2$ & Empresas diversificadas & 3.676 \\
\hline
\end{tabular}

Tabla 8. Desagregación de las empresas diversificadas.

\begin{tabular}{|l|c|c|c|}
\hline \multicolumn{1}{|c|}{ Dirección de la diversificación } & Índice & $\mathbf{N}^{\mathbf{0}}$ observaciones & Porcentajes \\
\hline Diversificación Relacionada & 1 & 1.526 & 42 \\
\hline Diversificación No Relacionada & 2 & 2.150 & 58 \\
\hline Total obs. empresas diversificadas & $1+2$ & 3.676 & 100 \\
\hline
\end{tabular}

Tabla 9. Distribución de empresas diversificadas por sectores industriales 1991-2005.

\begin{tabular}{|c|c|c|c|c|c|}
\hline $\begin{array}{l}\text { Códigos } \\
\text { CNAE }\end{array}$ & Sectores & $\begin{array}{c}\mathbf{N}^{\mathbf{o}} \\
\text { medio de } \\
\text { empresas } \\
\text { en el } \\
\text { sector }\end{array}$ & $\begin{array}{l}\mathbf{N}^{\circ} \text { medio de } \\
\text { empresas } \\
\text { diversificadas } \\
\text { en el sector }\end{array}$ & $\begin{array}{c}\text { Porcentaje } \\
\text { de empresas } \\
\text { diversificadas } \\
\text { en el sector }\end{array}$ & $\begin{array}{l}\text { Porcentaje de empresas } \\
\text { diversificadas en relación } \\
\text { con el resto de los } \\
\text { sectores }\end{array}$ \\
\hline 1 & Industria cárnica & 50 & 4 & 8 & 1,6 \\
\hline 2 & Prod. alimenticios y tabaco & 174 & 18 & 10,3 & 7,4 \\
\hline 3 & Bebidas & 36 & 3 & 8,3 & 1,2 \\
\hline 4 & Textiles & 180 & 21 & 11,7 & 8,6 \\
\hline 5 & Cuero y calzado & 54 & 3 & 5,6 & 1,2 \\
\hline 6 & Industria de la madera & 48 & 6 & 12,5 & 2,5 \\
\hline 7 & Papel & 51 & 7 & 13,7 & 2,9 \\
\hline 8 & Edición y artes gráficas & 91 & 9 & 9,9 & 3,7 \\
\hline 9 & Prod. químicos & 122 & 22 & 18,0 & 9 \\
\hline 10 & Prod. de caucho y plástico & 93 & 11 & 11,8 & 4,5 \\
\hline 11 & $\begin{array}{l}\text { Prod. de minerales no } \\
\text { metálicos }\end{array}$ & 122 & 13 & 10,7 & 5,3 \\
\hline 12 & Metalurgia & 57 & 11 & 19,3 & 4,5 \\
\hline 13 & Prod. Metálicos & 168 & 33 & 19,6 & 13,5 \\
\hline 14 & Maq. y equipo mecánico & 132 & 27 & 20,5 & 11,1 \\
\hline 15 & $\begin{array}{l}\text { Máquinas de oficina, equipos } \\
\text { informáticos, de proceso, } \\
\text { óptica y similares }\end{array}$ & 28 & 6 & 21,4 & 2,5 \\
\hline 16 & $\begin{array}{l}\text { Maquinaria y material } \\
\text { eléctrico y electrónico }\end{array}$ & 121 & 22 & 18,2 & 9 \\
\hline 17 & Vehículo de motor & 83 & 12 & 14,5 & 4,9 \\
\hline 18 & Otro material de transporte & 39 & 3 & 7,7 & 1,2 \\
\hline 19 & Muebles & 87 & 9 & 10,3 & 3,7 \\
\hline 20 & Otras inds. manufactureras & 39 & 4 & 10,3 & 1,6 \\
\hline \multicolumn{2}{|c|}{ Total observaciones de empresas } & 1.775 & 244 & 13,7 & 100 \\
\hline
\end{tabular}


Tabla 10. Distribución de empresas con diversificación relacionada y no relacionada por sectores industriales 19912005.

\begin{tabular}{|c|c|c|c|c|c|c|}
\hline $\begin{array}{l}\text { Códigos } \\
\text { CNAE }\end{array}$ & Sectores & $\begin{array}{c}\text { Número } \\
\text { medio de } \\
\text { empresas } \\
\text { diversificadas } \\
\text { en el sector }\end{array}$ & $\begin{array}{c}\text { Número } \\
\text { medio de } \\
\text { empresas con } \\
\text { diversificación } \\
\text { relacionada }\end{array}$ & $\begin{array}{c}\text { Porcentaje de } \\
\text { empresas con } \\
\text { diversificación } \\
\text { relacionada }\end{array}$ & $\begin{array}{l}\text { Número medio } \\
\text { de empresas con } \\
\text { diversificación } \\
\text { no relacionada }\end{array}$ & $\begin{array}{l}\text { Porcentaje de } \\
\text { empresas con } \\
\text { diversificación } \\
\text { no relacionada }\end{array}$ \\
\hline 1 & Industria cárnica & 4 & 2 & 50 & 2 & 50 \\
\hline 2 & $\begin{array}{l}\text { Prod. alimenticios y } \\
\text { tabaco }\end{array}$ & 18 & 17 & 94 & 1 & 6 \\
\hline 3 & Bebidas & 3 & 3 & 100 & 0 & 0 \\
\hline 4 & Textiles & 21 & 9 & 43 & 12 & 57 \\
\hline 5 & Cuero y calzado & 3 & 1 & 33 & 2 & 67 \\
\hline 6 & Industria de la madera & 6 & 3 & 50 & 3 & 50 \\
\hline 7 & Papel & 7 & 2 & 29 & 5 & 71 \\
\hline 8 & Edición y artes gráficas & 9 & 4 & 44 & 5 & 56 \\
\hline 9 & Productos químicos & 22 & 13 & 59 & 9 & 41 \\
\hline 10 & $\begin{array}{l}\text { Prod. de caucho y } \\
\text { plástico }\end{array}$ & 11 & 1 & 9 & 10 & 91 \\
\hline 11 & $\begin{array}{l}\text { Prod. de minerales no } \\
\text { metálicos }\end{array}$ & 13 & 6 & 46 & 7 & 54 \\
\hline 12 & Metalurgia & 11 & 4 & 36 & 7 & 64 \\
\hline 13 & Productos Metálicos & 33 & 13 & 39 & 20 & 61 \\
\hline 14 & $\begin{array}{l}\text { Maq. y equipo } \\
\text { mecánico }\end{array}$ & 27 & 9 & 33 & 18 & 67 \\
\hline 15 & $\begin{array}{l}\text { Máquinas de oficina, } \\
\text { equipos informáticos, } \\
\text { de proceso, óptica y } \\
\text { similares }\end{array}$ & 6 & 0 & 0 & 6 & 100 \\
\hline 16 & $\begin{array}{l}\text { Maquinaria y material } \\
\text { eléctrico y electrónico }\end{array}$ & 22 & 11 & 50 & 11 & 50 \\
\hline 17 & Vehículo de motor & 12 & 4 & 33 & 8 & 67 \\
\hline 18 & $\begin{array}{l}\text { Otro material de } \\
\text { transporte }\end{array}$ & 3 & 0 & 0 & 3 & 100 \\
\hline 19 & Muebles & 9 & 1 & 11 & 8 & 89 \\
\hline 20 & $\begin{array}{l}\text { Otras inds. } \\
\text { manufactureras }\end{array}$ & 4 & 1 & 25 & 3 & 75 \\
\hline \multicolumn{2}{|c|}{ Total observaciones de empresas } & 244 & 102 & 42 & 142 & 58 \\
\hline
\end{tabular}

A continuación, la tabla 11 presenta la distribución sectorial, basada en la clasificación CNAE-93, de la muestra de observaciones de empresas diversificadas seleccionada en comparación con la distribución poblacional de la ESEE para el período 1991-2005.

Como puede observarse en la tabla 11, las empresas industriales que operan en los siguientes sectores: Textil $(10,1 \%)$, productos alimenticios y tabaco $(9,8 \%)$, productos metálicos $(9,5 \%)$, maquinaria y equipo mecánico $(7,4 \%)$, productos químicos $(6,9 \%)$, productos de minerales no metálicos $(6,9)$ y maquinaria y material eléctrico y electrónico $(6,8 \%)$ presentan el mayor peso relativo en la población de observaciones de empresas industriales españolas. De la misma forma, al considerar la muestra de empresas industriales que operan en los siguientes sectores: Productos metálicos $(13,6 \%)$, maquinaria y equipo mecánico $(11 \%)$, productos químicos $(9,1 \%)$, maquinaria y material eléctrico y electrónico $(8,9 \%)$, textil $(8,7 \%)$, productos alimenticios y tabaco $(7,5 \%)$ y productos de minerales no metálicos $(5,2 \%)$ presentan el mayor peso relativo en la muestra de observaciones de empresas diversificadas españolas. Por esto, tanto en la muestra como en la población la representatividad de los sectores de mayor peso relativo es "similar" y sólo difiere en orden de importancia (de mayor a menor), si se consideran los porcentajes. 
Tabla 11. Distribución sectorial de las empresas de la muestra.

\begin{tabular}{|l|c|c|c|c|}
\hline \multirow{2}{*}{ Sectores } & \multicolumn{2}{c|}{ Muestra } & \multicolumn{2}{c|}{ ESEE } \\
\cline { 2 - 5 } & $\mathbf{N}$ & $\mathbf{\%}$ & $\mathbf{N}$ & $\mathbf{\%}$ \\
\hline 1. Industria cárnica & 61 & 1,7 & 744 & 2,8 \\
\hline 2. Productos alimenticios y tabaco & 275 & 7,5 & 2.609 & 9,8 \\
\hline 3. Bebidas & 43 & 1,2 & 534 & 2,0 \\
\hline 4. Textiles & 319 & 8,7 & 2.700 & 10,1 \\
\hline 5. Cuero y calzado & 44 & 1,2 & 805 & 3,0 \\
\hline 6. Industria de la madera & 91 & 2,5 & 719 & 2,7 \\
\hline 7. Papel & 98 & 2,7 & 763 & 2,9 \\
\hline 8. Edición y artes gráficas & 140 & 3,8 & 1.371 & 5,1 \\
\hline 9. Productos químicos & 335 & 9,1 & 1.824 & 6,9 \\
\hline 10. Productos de caucho y plástico & 168 & 4,6 & 1.396 & 5,2 \\
\hline 11. Productos de minerales no metálicos & 191 & 5,2 & 1.836 & 6,9 \\
\hline 12. Metalurgia & 166 & 4,5 & 858 & 3,2 \\
\hline 13. Productos Metálicos & 499 & 13,6 & 2.517 & 9,5 \\
\hline 14. Maquinaria y equipo mecánico & 406 & 11 & 1.980 & 7,4 \\
\hline 15. Máq. de oficina, equipos informáticos, de proceso, óptica y similares & 87 & 2,4 & 427 & 1,6 \\
\hline 16. Maquinaria y material eléctrico y electrónico & 329 & 8,9 & 1.822 & 6,8 \\
\hline 17. Vehículo de motor & 179 & 4,9 & 1.245 & 4,7 \\
\hline 18. Otro material de transporte & 50 & 1,3 & 587 & 2,2 \\
\hline 19. Muebles & 135 & 3,6 & 1.300 & 4,9 \\
\hline 20. Otras industrias manufactureras & 60 & 1,6 & 579 & 2,2 \\
\hline Total observaciones de empresas & $\mathbf{3 . 6 7 6}$ & $\mathbf{1 0 0}$ & $\mathbf{2 6 . 6 1 6}$ & $\mathbf{1 0 0}$ \\
\hline
\end{tabular}

Tabla 12. Cobertura de empresas según su tamaño.

\begin{tabular}{|l|c|c|c|c|c|c|c|c|}
\hline \multirow{2}{*}{ Sectores } & \multicolumn{4}{|c|}{ Muestra } & \multicolumn{4}{c|}{ ESEE } \\
\cline { 2 - 9 } & $\begin{array}{c}\text { Empresas } \mathbf{2 0 0} \\
\text { empleados }\end{array}$ & \multicolumn{2}{|c|}{$\begin{array}{c}\text { Empresas } \mathbf{2 0 0} \\
\text { empleados }\end{array}$} & \multicolumn{2}{|c|}{$\begin{array}{c}\text { Empresas } \\
\text { empleados }\end{array}$} & $\begin{array}{c}\text { Empresas } \mathbf{2 0 0} \\
\text { empleados }\end{array}$ \\
\cline { 2 - 9 } & $\mathbf{N}$ & $\mathbf{\%}$ & $\mathbf{N}$ & $\mathbf{\%}$ & $\mathbf{N}$ & $\mathbf{\%}$ & $\mathbf{N}$ & $\mathbf{\%}$ \\
\hline 1. Industria cárnica & 24 & 0,7 & 37 & 1,0 & 497 & 1,9 & 247 & 1 \\
\hline 2. Prod. alimenticios y tabaco & 132 & 3,6 & 143 & 4 & 1.809 & 6,8 & 799 & 3,0 \\
\hline 3. Bebidas & 37 & 1,0 & 6 & 0,2 & 262 & 1 & 272 & 1,0 \\
\hline 4. Textiles & 168 & 4,6 & 151 & 4,1 & 2.101 & 7,9 & 599 & 2,3 \\
\hline 5. Cuero y calzado & 42 & 1,1 & 2 & 0,1 & 774 & 3 & 31 & 0,1 \\
\hline 6. Industria de la madera & 86 & 2,3 & 5 & 0,1 & 603 & 2,3 & 116 & 0,4 \\
\hline 7. Papel & 69 & 1,9 & 29 & 0,8 & 458 & 1,7 & 305 & 1,1 \\
\hline 8. Edición y artes gráficas & 96 & 2,7 & 44 & 1,2 & 1.059 & 4 & 312 & 1,2 \\
\hline 9. Productos químicos & 165 & 4,5 & 170 & 4,6 & 886 & 3,3 & 938 & 3,5 \\
\hline 10. Prod. de caucho y plástico & 110 & 3 & 58 & 1,6 & 1.005 & 3,8 & 391 & 1,5 \\
\hline 11. Prod. de minerales no metálicos & 117 & 3,2 & 74 & 2,0 & 1.234 & 4,6 & 602 & 2,3 \\
\hline 12. Metalurgia & 57 & 1,5 & 109 & 3 & 366 & 1,4 & 492 & 1,8 \\
\hline 13. Productos metálicos & 373 & 10,1 & 126 & 3,4 & 1.992 & 7,5 & 525 & 2 \\
\hline 14. Maquinaria y equipo mecánico & 231 & 6,3 & 175 & 4,8 & 1.337 & 5,0 & 643 & 2,4 \\
\hline 15. Maq. Ofic., equipos informáticos, de proceso, óptica & & & & & & & & \\
y similares & 50 & 1,3 & 37 & 1,0 & 254 & 1 & 173 & 0,64 \\
\hline 16. Maquinaria y material eléctrico y electrónico & 192 & 5,2 & 137 & 3,7 & 1.123 & 4,2 & 699 & 2,6 \\
\hline 17. Vehículo de motor & 66 & 1,8 & 113 & 3,0 & 521 & 2 & 724 & 2,7 \\
\hline 18. Otro material de transporte & 33 & 0,9 & 17 & 0,5 & 314 & 1,2 & 273 & 1,0 \\
\hline 19. Muebles & 123 & 3,3 & 12 & 0,3 & 1.140 & 4,3 & 160 & 0,6 \\
\hline 20. Otras industrias manufactureras & 37 & 1,0 & 23 & 0,6 & 467 & 1,8 & 112 & 0,4 \\
\hline Total de observaciones & $\mathbf{2 . 2 0 8}$ & $\mathbf{6 0}$ & $\mathbf{1 . 4 6 8}$ & $\mathbf{4 0}$ & $\mathbf{1 8 . 2 0 2}$ & $\mathbf{6 9}$ & $\mathbf{8 . 4 1 4}$ & $\mathbf{3 1}$ \\
\hline
\end{tabular}


Tabla 13. Cobertura de empresas con diversificación relacionada y no relacionada según su tamaño.

\begin{tabular}{|c|c|c|c|c|c|c|c|c|}
\hline \multirow{3}{*}{ Sectores } & \multicolumn{4}{|c|}{ Muestra de empresas con DR } & \multicolumn{4}{|c|}{ Muestra de empresas con DNR } \\
\hline & \multicolumn{2}{|c|}{$\begin{array}{c}\text { Empresas } \leq 200 \\
\text { empleados }\end{array}$} & \multicolumn{2}{|c|}{$\begin{array}{c}\text { Empresas }>200 \\
\text { empleados }\end{array}$} & \multicolumn{2}{|c|}{$\begin{array}{c}\text { Empresas } \leq 200 \\
\text { empleados }\end{array}$} & \multicolumn{2}{|c|}{$\begin{array}{c}\text { Empresas }>200 \\
\text { empleados }\end{array}$} \\
\hline & $\mathbf{N}$ & $\%$ & $\mathbf{N}$ & $\%$ & $\mathbf{N}$ & $\%$ & $\mathbf{N}$ & $\%$ \\
\hline 1. Industria cárnica & 20 & 1,3 & 16 & 1,0 & 4 & 0,2 & 21 & 1 \\
\hline 2. Productos alimenticios y tabaco & 122 & 8 & 126 & 8,3 & 10 & 0,5 & 17 & 0,8 \\
\hline 3. Bebidas & 31 & 2,0 & 6 & 0,4 & 6 & 0,3 & 0 & 0 \\
\hline 4. Textiles & 60 & 4 & 67 & 4,4 & 108 & 5,0 & 84 & 4 \\
\hline 5. Cuero y calzado & 11 & 0,7 & 2 & 0,1 & 31 & 1,4 & 0 & 0 \\
\hline 6. Industria de la madera & 46 & 3,0 & 1 & 0,1 & 40 & 2 & 4 & 0,2 \\
\hline 7. Papel & 12 & 0,8 & 12 & 0,8 & 57 & 2,6 & 17 & 0,8 \\
\hline 8. Edición y artes gráficas & 42 & 2,8 & 15 & 1 & 54 & 2,5 & 29 & 1,3 \\
\hline 9. Productos químicos & 91 & 6 & 108 & 7,1 & 74 & 3,4 & 62 & 3 \\
\hline 10. Productos de caucho y plástico & 6 & 0,4 & 2 & 0,1 & 104 & 4,8 & 56 & 2,6 \\
\hline 11. Prod. de minerales no metálicos & 53 & 3,5 & 29 & 2 & 64 & 3 & 45 & 2,1 \\
\hline 12. Metalurgia & 7 & 0,5 & 47 & 3,1 & 50 & 2,3 & 62 & 3 \\
\hline 13. Productos metálicos & 157 & 10,3 & 32 & 2,1 & 216 & 10,0 & 94 & 4,4 \\
\hline 14. Maquinaria y equipo mecánico & 59 & 4 & 82 & 5,4 & 172 & 8 & 93 & 4,3 \\
\hline $\begin{array}{l}\text { 15. Máquinas de oficina, equipos informáticos, de } \\
\text { proceso, óptica y similares }\end{array}$ & 4 & 0,3 & 2 & 0,1 & 46 & 2,1 & 35 & 1,6 \\
\hline 16. Maq. y material eléctrico y electrónico & 107 & 7,0 & 57 & 3,7 & 85 & 4 & 80 & 3,7 \\
\hline 17. Vehículo de motor & 4 & 0,3 & 56 & 3,7 & 62 & 3 & 57 & 2,6 \\
\hline 18. Otro material de transporte & 2 & 0,1 & 1 & 0,1 & 31 & 1,4 & 16 & 0,7 \\
\hline 19. Muebles & 7 & 0,5 & 4 & 0,3 & 116 & 5,4 & 8 & 0,4 \\
\hline 20. Otras industrias manufactureras & 11 & 0,7 & 9 & 0,6 & 26 & 1,2 & 14 & 0,7 \\
\hline Total de observaciones & 852 & 56 & 674 & 44 & 1.356 & 63 & 794 & 37 \\
\hline
\end{tabular}

En cambio, las empresas industriales que operan en los siguientes sectores: Máquinas de oficina, equipos informáticos, de proceso, óptica y similares $(1,6 \%)$, bebidas $(2,0 \%)$, otro material de transporte $(2,2 \%)$, otras industrias manufactureras $(2,2 \%)$, industria de la madera $(2,7 \%)$, industria cárnica $(2,8 \%)$ y papel $(2,9 \%)$ presentan el menor peso relativo en la población de observaciones de empresas industriales españolas. De manera similar, al considerar la muestra las empresas industriales que operan en los sectores: Bebidas (1,2\%), cuero y calzado $(1,2 \%)$, otro material de transporte $(1,3 \%)$, otras industrias manufactureras $(1,6 \%)$, industria cárnica $(1,7 \%)$, el sector de máquinas de oficina, equipos informáticos, de proceso, óptica y similares $(2,4 \%)$ y la industria de la madera $(2,5 \%)$ presentan el menor peso relativo en la muestra de observaciones de empresas diversificadas españolas. De esta forma, tanto en la muestra como en la población la representatividad de los sectores de menor peso relativo es "casi similar".

Por otra parte, otro interés de investigación consistió en analizar el tamaño de las empresas de la muestra. Lo anterior se debe a la relevancia que posee la variable tamaño en los estudios de Dirección Estratégica y Economía Industrial, entre otras disciplinas. De hecho, el tamaño se ha considerado como variable de control en muchas investigaciones [5, 8, 17, 23, 41, 46, 48]. Por ello, en el estudio de las organizaciones el análisis del tamaño sigue concentrando el interés de los investigadores de diversas áreas, quienes investigan diferentes fenómenos pero vinculados al mismo concepto, prueba de ella son los recientes estudios de Cichello [6], Tsai [42], Beck, Demirgüç-Kunt y Maksimovic [1], Halkos y Tzeremes [11], Haan, Kisperska-Morón y Placzek [10], Pla-Barber y Alegre [32]. Sin embargo, pese a la relevancia que tiene el tamaño empresarial en todas las economías, la mayoría de las investigaciones se concentra en países desarrollados y no de reciente desarrollo, como el caso español.

Considerando lo anterior, el análisis de la diversificación de la empresa industrial española en función de su tamaño se encuentra en las tablas 12 y 13. Específicamente, la ESEE distingue el tamaño de las empresas según tengan más o menos de 200 trabajadores, por lo que, a la vista 
de la información disponible, este es el único criterio para discriminar entre pequeñas y grandes empresas. En la tabla 12 se presenta la cobertura de nuestra muestra de empresas diversificadas en relación con las consideradas por la ESEE, para los dos tipos de tamaño de la encuesta.

El número de empresas de más de doscientos trabajadores supone un $40 \%$ de la muestra, mientras que las pequeñas empresas representan un $60 \%$. Ello puede hacer suponer una cierta superioridad de las pequeñas empresas sobre las grandes en su proceso de diversificación, aunque también puede ser debido simplemente al mayor porcentaje (69) que representan las empresas de reducida dimensión en el conjunto de la ESEE. Un argumento para justificar esta superioridad numérica se puede deber, por una parte, a la ventaja que disfrutan las empresas de menor tamaño, en relación con las empresas de mayor dimensión, en cuanto a una mayor flexibilidad y coordinación de actividades que les permite tener una mayor capacidad para generar nuevos productos/servicios hacia otros mercados. Y, por otra parte, también se puede deber a la tendencia internacional que existe hacia la reestructuración organizacional o dowsizing seguido por las empresas. A diferencia de las grandes empresas que sufren deseconomías directivas [41], pese a que poseen bastantes recursos para llevar a cabo sus negocios, tales como capital, talentos directivos, entre otras [23].

En cuanto a la distribución sectorial por número de empleados, podemos destacar la concentración de empresas de menor dimensión en el sector de productos metálicos $(10,1 \%)$ y en el sector de maquinaria y equipo mecánico $(6,3 \%)$. Por el contrario, las empresas de mayor dimensión se localizan en el sector de maquinaria y equipo mecánico $(4,8 \%)$ y en el sector de productos químicos $(4,6 \%)$. La tabla 13 presenta una desagregación de la muestra para las empresas con diversificación relacionada y diversificación no relacionada, respectivamente, en función del tamaño de las mismas. Del conjunto de empresas que siguen diversificación relacionada, el $56 \%$ y el $44 \%$ corresponden a empresas pequeñas y grandes, respectivamente. De igual forma, del conjunto de empresas que siguen diversificación no relacionada, el $63 \%$ y el $37 \%$ corresponden a empresas pequeñas y grandes, respectivamente.

En cuanto a la distribución sectorial por número de empleados, se puede destacar la concentración de empresas de menor dimensión en el sector de productos metálicos $(10,3 \%$ y $10 \%)$ para ambas estrategias, a diferencia de las empresas de mayor dimensión que se ubican en el sector de productos alimenticios y tabaco $(8,3 \%)$ para la estrategia de diversificación relacionada y el sector de productos metálicos $(4,4 \%)$ para la estrategia de diversificación no relacionada.
Por último, como se puede apreciar, las empresas de menor tamaño, es decir, de menos de 200 trabajadores, son las que llevan a cabo un mayor porcentaje tanto de diversificación relacionada, como de diversificación no relacionada.

\section{CONCLUSIONES}

El presente trabajo intentó contribuir con la literatura existente, a partir de un breve análisis crítico de las diferentes formas de medidas sobre diversificación y a través de la propuesta de un índice de diversificación, construido a partir de la información disponible en la Encuesta sobre Estrategias Empresariales (ESEE).

Específicamente, se llevó a cabo un análisis de las principales formas de medir la diversificación empresarial, presentando las ventajas e inconvenientes que muestran cada una de ellas. De esta forma, se realiza un recorrido por las distintas medidas continuas y categóricas encontradas en la literatura especializada. A la vista de las limitaciones encontradas en algunas de ellas, se optó por construir un índice propio a partir de la información suministrada por la ESEE para un periodo de tiempo comprendido entre 1991 y 2005. Con este índice se pudo reconocer tanto si las empresas se especializan o se diversifican, así como la dirección de la diversificación seguida.

La aplicación de este índice a la realidad de la empresa industrial española permite deducir claramente que la línea predominante es la especialización, siendo relativamente escaso el número de empresas españolas que siguen procesos de diversificación. Además, de entre estas últimas se pueden discriminar aquellas que siguen diversificación relacionada y no relacionada, siendo levemente superiores aquellas que llevan a cabo diversificación no relacionada. Sin embargo, se ha argumentado que se puede deber a la forma en la cual hemos medido la diversificación, la cual pretende mostrar mayor robustez a la categoría de diversificación relacionada.

De la misma forma, se ha observado que tanto en la muestra como en la población la representatividad de los sectores de mayor peso relativo era "similar" y sólo difería en orden de importancia (de mayor a menor), si considerábamos los porcentajes. Al contrario, no sucedía lo mismo en el caso de los sectores de menor peso relativo.

Por último, se ha descubierto que la mayoría de las empresas que diversifican presentan un menor tamaño, es decir, menos de 200 empleados, independientemente de la dirección de diversificación elegida. La razón puede estar 
en la mayor flexibilidad y coordinación que presentan las empresas de menor tamaño sobre las de mayor dimensión en cuanto a estos dos atributos.

Sin embargo, también se debe considerar que el fenómeno de la reestructuración empresarial ha motivado "una vuelta" atrás al proceso de diversificación. Este fenómeno ha generado que los grandes conglomerados se deshagan de sus divisiones no relacionadas con sus negocios centrales, buscando una mayor relación con los negocios en los cuales participa. Este fenómeno puede haber afectado los resultados, razón por la cual se observa un mayor número de observaciones en la categoría de empresas especializadas. A la vez, la reestructuración de la cartera de negocios también ha ido acompañado de cambios organizacionales y, dentro de ellos, una tendencia a la reducción de plantillas o dowsizing, lo cual, en cierta forma, justifica que exista una mayor cantidad de empresas de menor dimensión en la realidad de las empresas industriales españolas. En resumen, se puede concluir que estos cambios a nivel de negocios y a nivel organizacional, que han sufrido las empresas multidivisionales a nivel internacional, se han dejado sentir, de forma similar, en la realidad de las empresas españolas y se comprueba a través de los resultados antes presentados.

\section{AGRADECIMIENTOS}

Los autores quieren agradecer a la Fundación SEPI en España, por la colaboración a través de la base de datos proporcionada, ESEE. Y, además, agradecen a la Universidad del Bío-Bío, por su apoyo a través del proyecto $0848162 / \mathrm{R}$.

\section{REFERENCIAS}

[1] T. Beck, A. Demirgüç-Kunt and V. Maksimovic. "The Influence of Financial and Legal Institutions on Firm Size". Journal of Banking \& Finance. Vol. 30, pp. 2995-3015. 2006.

[2] B. Boyd, S. Gove and M. Hitt. "Consequences of Measurement Problems in Strategic Management Research: The Case of Amihud and Lev". Strategic Management Journal. Vol. 26, pp. 367-375. 2005.

[3] E. Bueno. "Dirección Estratégica de la Empresa. Metodología, Técnicas y Casos". Pirámide. $5^{\text {a }}$ Edición. Madrid, España. 1996.
[4] E. Bueno, P. Morcillo e I. De Pablo. "Dimensiones Competitivas de la Empresa Española". Papeles de Economía Española. № 39, pp. 37-65. 1989.

[5] A. Chakrabarti, K. Singh and I. Mamad. "Diversification and Performance: Evidence From East Asian Firms". Strategy Management Journal. Vol. 28, pp. 101-120. 2007.

[6] M. Cichello. "The Impact of Firm Size on PayPerformance Sensitivities". Journal of Corporate Finance. Vol. 11, pp. 609-627. 2005.

[7] E. Døving and P. Gooderham. "Dynamic capabilities as antecedents of the scope of related diversification: The case of small firm accountancy practices". Strategy Management Journal. Vol. 29, pp. 841857. 2008.

[8] M. Goranova, T. Alessandri, P. Brandes and R. Dharwadkar. "Managerial Ownership and Corporate Diversification: A Longitudinal View". Strategy Management Journal. Vol. 28, pp. 211225. 2007.

[9] R. Grant and A. Jammine. "Performance Differences between the Wrigley/Rumelt Strategic Categories". Strategic Management Journal. Vol. 9, pp. 333-346. 1988.

[10] J. Haan, D. Kisperska-Morón and E. Placzek. "Logistics Management and Firm Size; A Survey Among Polish Small And Medium Enterprises". International Journal of Production Economics. Vol. 108, pp. 119-126. 2007.

[11] G. Halkos and N. Tzeremes. "Productivity Efficiency and Firm Size: An Empirical Analysis of Foreign Owned Companies". International Business Review. Vol. 16, pp. 713-731. 2007.

[12] Caron H. St. John and Ernest H. Hall, Jr. "A Methodological Note on Diversity Measurement". Strategic Management Journal. Vol. $15 \mathrm{~N}^{\mathrm{o}} 2$, pp. 153-168. 1994.

[13] R. Hoskisson and M. Hitt. "Antecedents and Performance Outcomes of Diversification: A Review and Critique of Theoretical Perspectives". Journal of Management. Vol. 16, pp. 461-509. 1990.

[14] R. Hoskisson, M. Hitt, R. Johnson and D. Moesel. "Construct Validity of an Objetive (Entropy) 
Categorical Measure of Diversification Strategy". Strategic Management Journal. Vol. 14, pp. 215235. 1993.

[15] P. Huerta y J. Navas. "Grado y Dirección de la Diversificación de las Empresas Industriales Españolas: Un Análisis de la Estrategia de Diversificación Relacionada”. Ingeniare. Revista chilena de ingeniería. Vol. $14 \mathrm{~N}^{\mathrm{o}}$ 3, pp. 213-228. 2006.

[16] P. Huerta y J. Navas. "Análisis de la relación entre la diversificación y los resultados empresariales: Una aplicación teórica”. Análisis Económico. Vol. 22 No 49, pp. 133-148. 2007.

[17] C. Hull and S. Rothenberg. "Firm Performance: the Interactions of Corporate Social Performance With Innovation and Industry Differentiation". Strategy Management Journal. Vol. 29, pp. 781789. 2008.

[18] A. Jacquemin and C. Berry. "Entropy Measure of Diversification and Corporate Growth". The Journal of Industrial Economics. Vol. $27 \mathrm{~N}^{\circ} 4$, pp. 359-369. 1979.

[19] W. Kim. "Developed A Global Diversification Measure”. Management Science. Vol. $35 \mathrm{~N}^{\mathrm{o}} 3$, pp. 376-385. 1989.

[20] M. Lubatkin. "Mergers Strategies and Stockholder Value". Strategic Management Journal. Vol. 8, pp. 39-53. 1987.

[21] C. Markides and P. Williamson. "Corporate Diversification and Organizational Structure: A Resource-Based View". Academy of Management Journal. Vol. 39 No 2, pp. 340-367. 1996.

[22] F. Merino De Lucas y D. Rodríguez Rodríguez. "Diversificación y Tamaño en las Empresas Industriales Españolas”. Papeles de Economía Española. No 78-79, pp. 236-249. 1999.

[23] L. Mesquita and S. Lazzarini. "Horizontal and Vertical Relationships in Developing Economies. Implications for Smes'Access to Global Markets". Academy of Management Journal. Vol. $51 \mathrm{~N}^{\mathrm{o}} 2$, pp. 359-380. 2008.

[24] D. Miller. “Technological Diversity, Related Diversification, and Firm Performance”. Strategy
Management Journal. Vol. 27, pp. 601-619. 2006.

[25] C. Montgomery. "The Measure of Firm Diversification: Some New Empirical Evidence". Academy of Management Journal. Vol. $25 \mathrm{~N}^{\mathrm{o}} 2$, pp. 299-307. 1982.

[26] C. Montgomery. "Product-Market Diversification and Market Power". Academy of Management Journal. Vol. 8, pp. 789-798. 1985.

[27] K. Palepu. "Diversification Strategy, Profit Performance and the Entropy Measure". Strategic Management Journal. Vol. 6, pp. 239-255. 1985.

[28] C. Park. "The Effects of Prior Performance on the Choice Between Related and Unrelated Acquisitions: Implications For The Performance Consequences of Diversification Strategy". Journal of Management Studies. Vol. 39 N $^{\circ}$ 7, pp. 1003-1019. 2002.

[29] A. Pehrsson. "Business Relatedness and Performance: A Study of Managerial Perceptions". Strategic Management Journal. Vol. 27, pp. 265-282. 2006.

[30] M. Pérez. "Problemas Vinculados con la Diversificación Empresarial: La Medición de la Estrategia de la Empresa”. Cuadernos de Estudios Empresariales. No 8, pp. 195-213. 1998.

[31] R. Pitts and H. Hopkins. "Firm Diversity: Conceptualization and Measurement", Academy Of Management Review. Vol. 7 No 4, pp. 620-629. 1982.

[32] J. Pla-Barber and J. Alegre. "Analysing the Link between Export Intensity, Innovation and Firm Size in a Science-Based Industry". International Business Review. Vol. 16, pp. 275-293. 2007.

[33] V. Ramanujan and P. Varadarajan. "Research on Corporate Diversification: A Synthesis". Strategic Management Journal. Vol. 10, pp. 523-551. 1989.

[34] R. Reed. "Bimodality in Diversification", Managerial and Decision Economics. Vol. 2, pp. 57-66. 1991.

[35] R. Reed and J. Sharp. "Confirmation of the Specialization Ratio". Applied Economics. Vol. 19, pp. 393-405. 1987. 
[36] R. Rumelt. "Strategy, Structure, And Economic Performance". Harvard Business School Press. Boston. 1974.

[37] R. Rumelt. "Diversification Strategy and Profitability". Strategic Management Journal. Vol. 3, pp. 359-369. 1982.

[38] R. Sambharya. "Assessing the Construct Validity of Strategic and Sic-Based Measures of Corporate Diversification". British Journal of Management. Vol. 11, pp. 163-173. 2000.

[39] C. Snow and D. Hambrick. "Measuring Organizational Strategies: Some Theoretical and Methodological Issues". Academy of Management Review. Vol. 5, pp. 527-538. 1980.

[40] I. Suárez. "Estrategia de Diversificación y Resultados de la Empresa Española”. Revista de Economía Aplicada. Vol. 11 No 4, pp. 103-128. 1994.

[41] H. Tanriverdi and C. Lee. "Within-Industry Diversification and Firm Performance in the Presence of Network Externalities: Evidence From The Software Industry". Academy of Management Journal. Vol. 51 No 2, pp. 381-397. 2008.

[42] K. Tsai. "R \& D Productivity and Firm Size: An Nonlinear Examination”. Technovation. Vol. 25. pp. 795-803. 2005.
[43] L. Valenzuela, J. García and M. Blasco. "Orientación al valor del cliente y las nuevas métricas de marketing. Revisión y análisis". Panorama Socioeconométrico. No 34, pp. 70-75. 2007.

[44] P. Varadarajan and V. Ramanujam. "Diversification and Performance: A Reexamination Using A New Two-Dimensional Conceptualization of Diversifity In Firm". Academy of Management Journal. Vol. $30 \mathrm{~N}^{\mathrm{o}} 2$, pp. 380-393. 1987.

[45] N. Venkatraman and J. Grant. "Construct Measurement in Organizational Strategy Research. A Critique And Proposal". Academy of Management Review. Vol. 11, pp. 71-87. 1986.

[46] M. Wiersema and H. Bowen. "Corporate Diversification: The Impact of Forein Competition, Industry Globalization, and Product Diversification". Strategy Management Journal. Vol. 29, pp. 115132. 2008.

[47] L. Wrigley. "Divisional Autonomy and Diversification". Tesis Doctoral no Publicada. Harvard Business School, Boston. 1970.

[48] C. Zott and R. Amit. "The Fit between Product Market Strategy and Business Model: Implications for Firm Performance". Strategy Management Journal. Vol. 29, pp. 1-26. 2008. 